\title{
SOME SPECTRAL PROPERTIES OF THE PERTURBED POLYHARMONIC OPERATOR
}

\author{
DANIEL EIDUS
}

\begin{abstract}
We deal with the polyharmonic operator perturbed by a potential, decreasing at infinity as $|x|^{-\sigma}$. Under some conditions we obtain the absence of eigenvalues in a neighbourhood of the point $z=0$, the existence of the strong limit and the asymptotic expansion of the corresponding resolvent $R_{z}$, considered in weighted $L^{2}$-spaces, as $z \rightarrow 0$, where $z$ is the spectral parameter.
\end{abstract}

Let us consider the operator

$$
L=(-\Delta)^{m}+q(x)
$$

in the space $L^{2}\left(\mathbf{R}^{n}\right)$, where $n$ is odd,

$$
2 m>n,
$$

$\operatorname{Im} q=0, q$ is Lebesgue measurable on $\mathbf{R}^{n}$, a.e. on $\mathbf{R}^{n}$,

$$
|q| \leq c(1+|x|)^{-\sigma}
$$

$$
\sigma>4 m-n,
$$

and $c$ does not depend on $x$. The operator $L$ defined on the Sobolev space $H_{2 m}\left(\mathbf{R}^{n}\right)$ is selfadjoint. Let $Q$ be the set of functions $h$, defined on $\mathbf{R}^{n}$, each of them vanishing a.e. outside a sphere depending on $h$. It is known [3] that if

$$
q \in Q
$$

then the resolvent kernel $G(x, y, k)$ of $L$, where

$$
k=z^{1 / 2 m} \in S_{m}:=\{k: k \in \mathbf{C}, 0<\arg k<\pi / m\},
$$

has a meromorphic continuation onto the whole $\mathbf{C}$. In the special case, where $q=0$ on $\mathbf{R}^{n}$ and (2) is valid, the point $k=0$ is a pole of $G$. But if a.e. on $\mathbf{R}^{n}$,

$$
q \geq 0
$$

$$
\operatorname{meas}\left\{x: x \in \mathbf{R}^{n}, q(x)>0\right\}>0
$$

and (5) is valid, then [1] $k=0$ is a regular point of $G$. We shall investigate the behaviour of $R_{z}$ as $z \rightarrow 0$ in the case where (5) does not hold, and therefore $G$ in general has no analytical continuation outside $S_{m}$. The results obtained below

Received by the editors January 15, 1985.

1980 Mathematics Subject Classification. Primary 35P05; Secondary 35J40. 
may be applied to the asymptotics as $t \rightarrow \infty$ of solutions of the corresponding nonstationary problems (see e.g. [2]).

Let $\phi$ be the class of functions $v \in H_{2 m}^{\text {loc }}$ satisfying the following conditions as $\rho \rightarrow \infty$ :

$$
\int_{|x|=\rho}\left|D^{j} v\right|^{2} d x=O\left(\rho^{2 \kappa}\right),
$$

where $D^{j}$ is any derivative of order $|j|, 0 \leq|j| \leq 2 m-1$, and

$$
\kappa=m-|j|-1 \text {. }
$$

Condition (U). The equation

$$
(-\Delta)^{m} v+q(x) v=0
$$

has only the trivial solution in the class $\phi$.

It is known that (2), (6), (7) imply (U). Denote by $N_{\varepsilon}$ the circle $|k|<\varepsilon$ in $\mathrm{C}$ and set

$$
N(\varepsilon)=N_{\varepsilon} \cap S_{m}, \quad N^{+}(\varepsilon) \cup(0, \varepsilon),
$$

where $(0, \varepsilon)$ is the interval $0<k<\varepsilon$. Let $L_{s}^{2}$ be the space of functions, $\varphi$, defined on $\mathbf{R}^{n}$, with the norm

$$
\|\varphi\|_{s}^{2}=\int(1+|x|)^{s}|\varphi|^{2} d x
$$

(we integrate over $\mathbf{R}^{n}$ ), and let $B_{s}$ be the normed space of bounded operators $A: L_{s}^{2} \rightarrow L_{-s}^{2}$, where $s>0$.

THEOREM 1. Let conditions (U), (2), (3), (4) be satisfied. Then there exists $\varepsilon>0$ such that

(i) There are no eigenvalues of $L$ in the interval $\left(-\varepsilon^{2 m}, \varepsilon^{2 m}\right)$.

(ii) For every

$$
s=4 m-n+\delta,
$$

where $\delta>0$, it is possible to find a constant $c_{s}>0$ so that the inequality

$$
\left\|R_{z}\right\|_{B_{s}} \leq c_{s}
$$

holds for each $k \in N(\varepsilon)$, where $z=k^{2 m}$.

PROOF. Choose some function $q_{0}(x)$ continuous on $\mathbf{R}^{n}$ and satisfying conditions (5), (6), (7), and set $L_{0}=(-\Delta)^{m}+q_{0}$. It is known [1] that the corresponding resolvent kernel $G_{0}(x, y, k)$ is continuous in some domain $\mathbf{R}^{n} \times \mathbf{R}^{n} \times N_{\varepsilon^{\prime}}, \varepsilon^{\prime}>0$, holomorphic on $N_{\varepsilon^{\prime}}$ in $k$ and for $x, y \in \mathbf{R}^{n}, k \in N\left(\varepsilon^{\prime}\right)$,

$$
\left|G_{0}(x, y, k)\right| \leq c(X Y)^{2 m-n},
$$

where $X=1+|x|, Y=1+|y|$, and $c$ does not depend on $x, y, k$. Choose some $\delta>0$ such that

$$
\delta<2(\sigma-(4 m-n)) .
$$

Suppose (i) is not valid. Then there exists a sequence $z_{l}=k_{l}^{2 m} \rightarrow 0, k_{l} \in N^{+}\left(\varepsilon^{\prime}\right)$, and a sequence $v_{l} \in H_{2 m}\left(\mathbf{R}^{n}\right)$ so that

$$
\left\|v_{l}\right\|_{-s}=1
$$


where $s$ is defined by (11), (14), and a.e. on $\mathbf{R}^{n}$,

$$
v_{l}=\int G_{0}(x, y, k) q_{1}(y) v_{l}(y) d y
$$

where $q_{1}=q_{0}-q$. Since (3), (11), (14), (15), (16) a.e. on $\mathbf{R}^{n}$,

$$
\left|v_{l}\right| \leq c X^{2 m-n}
$$

$l=1,2, \ldots$. Because of $(13),(15),(16),(17)$, there exists a subsequence $v_{l_{j}}$ converging in $L_{-s}^{2}$ to $v$, and a.e. on $\mathbf{R}^{n}$,

$$
v(x)=\int G_{0}(x, y, 0) q_{1}(y) v(y) d y .
$$

It follows from (18) (see [1]) that $v \in \phi$. Because of $(10),(\mathrm{U})$ we conclude that $v=0$ a.e. on $\mathbf{R}^{n}$. This contradicts (15); therefore (i) is proved. In order to prove (ii), suppose there exist some $s$, defined by (11) and (14), $f \in L_{s}^{2}$ and a sequence $k_{l} \rightarrow 0$, $k_{l} \in S_{m}$, so that $\left\|u_{l}\right\|_{-s} \rightarrow \infty$, where $u_{l}=R_{z_{l}} f, z_{l}=k_{l}^{2 m}$. Set $v_{l}=\left\|u_{l}\right\|_{-s}^{-1} u_{l}$. As above we obtain $v_{l_{j}} \rightarrow 0$ in $L_{-s}^{2}$, which contradicts (15). So the proof is complete.

COROLLARY. Let the conditions of Theorem 1 be satisfied. Then the strong limit $\rho$ of $R_{z}: L_{s}^{2} \rightarrow L_{-s}^{2}$ does exist, where $s$ is defined by (11), as $z \rightarrow 0, \operatorname{Im} z \neq 0$. Moreover, $\rho f \in \phi$ for any $f \in L_{s}^{2}$.

The next theorem immediately follows from Theorem 1 and the results of $M$. Murata [2, Theorems 8.7, 8.9, 8.10].

THEOREM 2. Let conditions (U), (2), (3) be satisfied, where $\sigma>4 m-n+2 l+2$, $l \geq 0$ is an integer. Then for each number $s>4 m-n+2 l+2$ there exist operators $\rho_{j} \in B_{s}, j=0,1, \ldots, l$ such that

$$
R_{z}=\sum_{j=0}^{l} \rho_{j} k^{j}+g(k) k^{l},
$$

where $z=k^{2 m}, k \in S_{m}$, and $\|g(k)\|_{B_{s}} \rightarrow 0$ as $k \rightarrow 0$. Moreover if $j / 2 m$ is not an integer, then $\rho_{j}$ is a finite rank operator.

\section{REFERENCES}

1. D. Eidus, Solutions of external boundary problems for small values of the spectral parameter, Integral Equations and Operator Theory 9 (1986), 21-32.

2. M. Murata, Asymptotic expansion in time for solutions of Schrödinger-type equations, J. Funct. Anal. 49 (1982), 10-56.

3. B. R. Vainberg, On exterior elliptic problems polynomially depending on a spectral parameter, Mat. Sb. 21 (1973), 221-239.

SChool of Mathematical Sciences, Tel-Aviv University, Ramat-Aviv, IsRael 